\title{
First-principles study on oxidation of Ge and its interface electronic structures
}

\author{
Tomoya Ono ${ }^{1,2 *}$, Shoichiro Saito ${ }^{3}$, and Shigeru Iwase ${ }^{4}$ \\ ${ }^{1}$ Center for Computational Sciences, University of Tsukuba, Tsukuba, Ibaraki 305-8577, Japan \\ 2 JST-PRESTO, Kawaguchi, Saitama 332-0012, Japan \\ ${ }^{3}$ Nitto Denko Corporation, Ibaraki, Osaka 567-8680, Japan \\ ${ }^{4}$ Graduate School of Engineering, Osaka University, Suita, Osaka 565-0871, Japan
}

\begin{abstract}
We review a series of first-principles studies on the defect generation mechanism and electronic structures of the $\mathrm{Ge} / \mathrm{GeO}_{2}$ interface. Several experimental and theoretical studies proved that $\mathrm{Si}$ atoms at the $\mathrm{Si} / \mathrm{SiO}_{2}$ interface are emitted to release interface stress. In contrast, total-energy calculation reveals that Ge atoms at the $\mathrm{Ge} / \mathrm{GeO}_{2}$ interface are hardly emitted, resulting in the low trap density. Even if defects are generated, those at the $\mathrm{Ge} / \mathrm{GeO}_{2}$ interface are found to behave differently from those at the $\mathrm{Si} / \mathrm{SiO}_{2}$ interface. The states attributed to the dangling bonds at the $\mathrm{Ge} / \mathrm{GeO}_{2}$ interface lie below the valence-band maximum of $\mathrm{Ge}$, while those at the $\mathrm{Si} / \mathrm{SiO}_{2}$ interface generate the defect state within the band gap of Si. First-principles electron-transport calculation elucidates that this characteristic behavior of the defect states is relevant to the difference in the leakage current through the $\mathrm{Si} / \mathrm{SiO}_{2}$ and $\mathrm{Ge} / \mathrm{GeO}_{2}$ interfaces.
\end{abstract}

\section{Introduction}

The impressive increase in the performance of microelectronic devices has been achieved by the continuous decrease in the length scale, which is approaching its technological and physical limits. However, it is becoming increasingly difficult to enhance the performance of complementary metal-oxide-semiconductor (MOS) devices through scaling based on conventional Si-based techniques. A considerable number of studies have been conducted to find alternative materials to further increase the performance of MOS field-effect transistors. Ge is considered as one of the best channel materials owing to its high intrinsic carrier mobility. Although the first bipolar transistor developed by Bardeen, Brattain, and Shockley consists of $\mathrm{Ge} / \mathrm{GeO}_{2}$, interest on $\mathrm{Ge}$-based devices has decreased with the advancement of $\mathrm{Si} / \mathrm{SiO}$-based transistors. This is because $\mathrm{Ge} / \mathrm{GeO}_{2}$ interfaces have generally been considered to include more defects than $\mathrm{Si} / \mathrm{SiO}_{2}$ interfaces, wherein the interface trap density typically lies in the range of the latter half of $10^{11}-10^{12} \mathrm{~cm}^{-2} \mathrm{eV}^{-1}$. ${ }^{1)}$ It is a general consensus that the formation 
of MOS interfaces with superior properties is the key issue to be resolved, and the interface properties are closely relevant to interface trap density. Thus, the understanding and control of the defects at the $\mathrm{Ge} / \mathrm{GeO}_{2}$ interface are important to enhance the performance of Ge-based devices because the $\mathrm{Ge} / \mathrm{GeO}_{2}$ interface exists even in $\mathrm{Ge} /$ high- $k$ oxide interfaces.

In the last decade, a considerable number of studies have been made on the fabrication of high-quality $\mathrm{Ge} / \mathrm{GeO}_{2}$ interfaces, and the low interface trap density in the $\mathrm{Ge} / \mathrm{GeO}_{2}$ interface can be achieved using a cap layer, ${ }^{2)}$ high-pressure thermal oxidation, ${ }^{3,4)}$ or ozone oxidation. ${ }^{5)}$ In 2008, Matsubara et al. ${ }^{6)}$ reported that the minimum interface trap density of lower than $10^{11} \mathrm{~cm}^{-2} \mathrm{eV}^{-1}$, which is lower than that in typical $\mathrm{Si} / \mathrm{SiO}_{2}$ interfaces without any hydrogen forming gas annealings, can be obtained for the $\mathrm{Ge} / \mathrm{GeO}_{2}$ interface fabricated by the dry oxidation without any hydrogen passivation treatment. In the following year, Hosoi et al. ${ }^{7}$ fabricated a high-quality $\mathrm{Ge} / \mathrm{GeO}_{2}$ interface by the conventional thermal treatment of Ge substrates. In addition, Lee et al. ${ }^{4)}$ also reported that the electrical properties of metal $/ \mathrm{GeO}_{2} / \mathrm{Ge}$ capacitors fabricated by the high-pressure oxidation of Ge are improved and the interface state density is reduced to $2 \times 10^{11} \mathrm{~cm}^{-2} \mathrm{eV}^{-1}$ near the middle of the valence-band maximum (VBM) and conduction-band minimum (CBM) of Ge even when post-deposition annealing is carried out. On the theoretical side, the structural and electronic properties of the $\mathrm{Ge} / \mathrm{GeO}_{2}$ interface have been investigated by first-principles calculations based on the density functional theory ${ }^{8,9)}$ and/or other methods avoiding the band gap problem in the local density approximation in the density functional theory. Houssa et al. ${ }^{10)}$ simulated the density of Ge-DBs at the $\mathrm{Ge} / \mathrm{GeO}_{2}$ interface as a function of oxidation temperature, by combining the viscoelastic data of $\mathrm{GeO}_{2}$ and the modified Maxwell's model, and claimed that the density of Ge-DBs is less than that of Si-DBs. The present authors found that hardly any defects are generated at the $\mathrm{Ge} / \mathrm{GeO}_{2}$ interface compared with the number generated at the $\mathrm{Si} / \mathrm{SiO}_{2}$ interface from the formation energy of defects at the $\mathrm{Ge} / \mathrm{GeO}_{2}$ interface. ${ }^{11)}$ In addition, Weber et al. ${ }^{12)}$ and Broqvist et al. ${ }^{13)}$ investigated the electronic structure of the defects in Ge bulk, which are expected to affect the interface properties, by the $G_{0} W_{0}$ approximation ${ }^{14)}$ and the hybrid functional method, ${ }^{15)}$ respectively. They reported the different behaviors of the dangling bonds (DBs) at the $\mathrm{Ge} / \mathrm{GeO}_{2}$ interface from those at the $\mathrm{Si} / \mathrm{SiO}_{2}$ interface.

In this article, we review the first-principles studies on the oxidation of Ge and its interface electronic structures together with relevant experimental results. In Sect. 2, studies on the interface atomic structures as well as defects are introduced in comparison with the case of Si. The simulations for the interface electronic structure and gate leakage current are reported in Sect. 3. Finally, our summary is given in Sect. 4. 
(a)
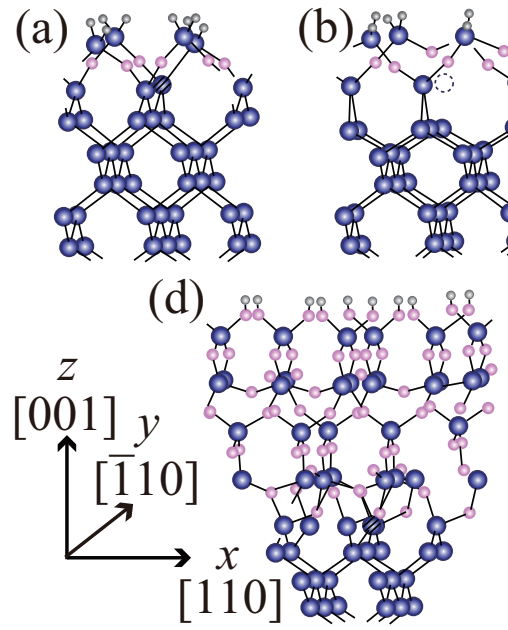

(b) 898
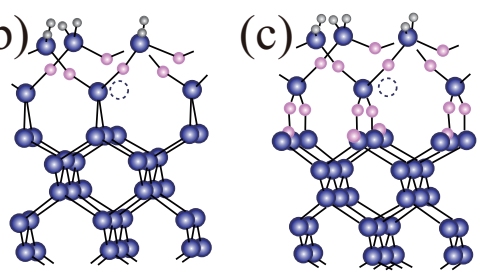

(e)

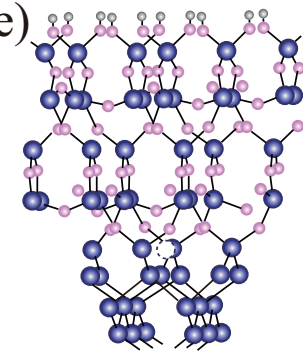

Fig. 1. (Color online) Atomic configurations of models. (a) Surface model where no Ge atom is emitted from the structure that includes three $\mathrm{O}$ atoms, (b) surface model where one Ge atom per unit cell is emitted from the structure including three $\mathrm{O}$ atoms, and (c) surface model where one Ge atom per unit cell is emitted from the structure including six $\mathrm{O}$ atoms. Interface models in which three additional $\mathrm{O}$ atoms are inserted into the $\mathrm{Ge} / \mathrm{GeO}_{2}$ interface (d) before emission and (e) after emission. The gray (light small), blue (dark large), and pink (light middle) balls are $\mathrm{H}, \mathrm{Ge}$, and $\mathrm{O}$ atoms, respectively. The hatched circles are the $\mathrm{Ge}$ atom being emitted and the broken circles indicate the position where the Ge atom is emitted.

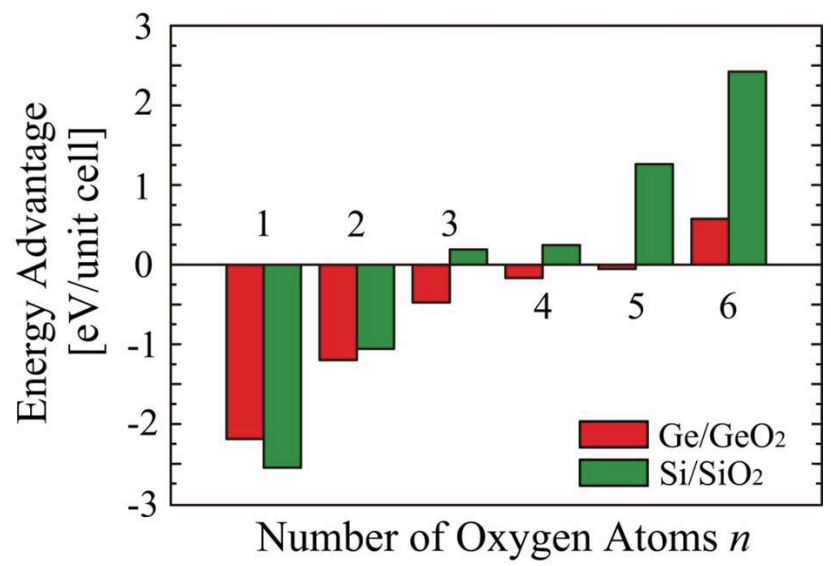

Fig. 2. (Color online) Energy advantage of Ge- and Si-emitting structures compared with nonemitting structures, as a function of the number of $\mathrm{O}$ atoms per unit cell $n$. Reprinted with modification with permission from Ref. 11. Copyright 2009 AIP Publishing LLC.

\section{Interface atomic structure}

\subsection{Atom emission from the interface}

The structural and electronic properties of $\mathrm{Ge} / \mathrm{GeO}_{2}$ interfaces have been investigated by several groups. ${ }^{10,11,16-24)}$ Although the residual order at $\mathrm{Si} / \mathrm{SiO}_{2}$ interfaces has been observed by transmission electron microscopy, ${ }^{25)}$ that at $\mathrm{Ge} / \mathrm{GeO}_{2}$ interfaces has not. Some studies ${ }^{18,23)}$ 


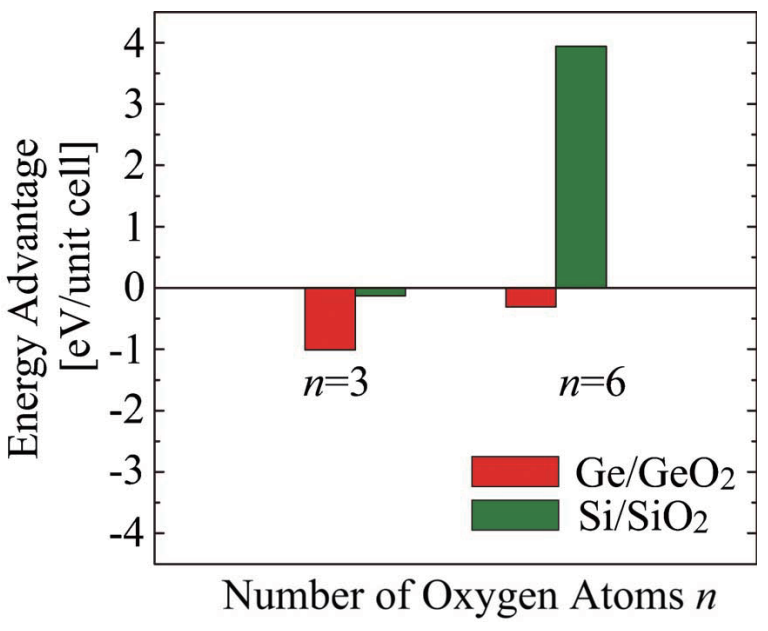

Fig. 3. (Color online) Energy advantage of Ge- and Si-emitting structures compared with nonemitting structures in the case of interface.

use the models in which amorphous $\mathrm{GeO}_{2}$ is attached to the $\mathrm{Ge}$ substrate to investigate electronic structures; however, it is not easy to derive definite conclusions using the models employing amorphous $\mathrm{GeO}_{2}$ because the model size treated by first-principles calculations is small to eliminate the effect of artificial periodic boundary conditions. Therefore, many studies $^{10,11,16,17,19-22,24)}$ adopt the interface atomic structures based on their $\mathrm{Si} / \mathrm{SiO}_{2}$ counterparts, ${ }^{26)}$ where crystalline $\mathrm{GeO}_{2}$ is attached to a Ge substrate.

Houssa et al. ${ }^{10)}$ simulated the density of $\mathrm{Ge}-\mathrm{DBs}$ at the $\mathrm{Ge} / \mathrm{GeO}_{2}$ interface as a function of oxidation temperature, by combining the viscoelastic data of $\mathrm{GeO}_{2}$ and the modified Maxwell's model, and claimed that the density of Ge-DBs is less than that of Si-DBs. Their results are in good agreement with the experimental results. ${ }^{4,6,7)}$ However, the formation mechanism of defects in the $\mathrm{Ge} / \mathrm{GeO}_{2}$ interface region during the oxidation is not well known.

The oxidation of the $\mathrm{Si} / \mathrm{SiO}_{2}$ interface was simulated by Kageshima and Shiraishi ${ }^{27,28)}$ using first-principles calculations and they found that a quartz phase can be obtained if Si atoms are kicked out from the interface during oxidation. Later, using the larger model, Yamasaki et $a .^{29)}$ also demonstrated that the $\mathrm{Si}$-atom emission from the $\mathrm{Si} / \mathrm{SiO}_{2}$ interface occurs and $P_{b}$-type defects are formed during oxidation. Moreover, the experiment using high-resolution Rutherford backscattering spectroscopy assured the Si-atom emission. ${ }^{30)}$ In the case of the oxidation of $\mathrm{Ge}$, it is reported that the volatilization of $\mathrm{GeO}$ from $\mathrm{Ge}$ atoms driven by the reaction at the $\mathrm{Ge} / \mathrm{GeO}_{2}$ interface is the origin of the deterioration of the interface. ${ }^{2)}$ In addition, the interface quality can be improved by suppressing the out-diffusion of $\mathrm{GeO}$ to the air 
using a cap layer on the top of $\mathrm{GeO}_{2}$. Therefore, the Ge-atom emission from the interface is strongly relevant to realize the low trap density in the $\mathrm{Ge} / \mathrm{GeO}_{2}$ interface.

The probability of the Ge-atom emission from the $\mathrm{Ge} / \mathrm{GeO}_{2}$ interface is compared with that from the $\mathrm{Si} / \mathrm{SiO}_{2}$ interface in terms of the total-energy calculation. ${ }^{11)}$ The calculations are performed on the basis of the density functional theory using the RSPACE code. ${ }^{31-34)}$ Figure 1 shows examples of the $\mathrm{Ge}(100)$ surface models. O atoms are sequentially inserted between Ge-Ge bonds from the surface, assuming atomic layer-by-layer oxide growth, and finally six $\mathrm{O}$ atoms are introduced into the unit cell. In the case of the oxidation of $\mathrm{Si}$, the first-principles calculations revealed that the $\mathrm{Si}$ atom in the second layer will be emitted to release the interface stress. ${ }^{27,28)}$ The example for the case of $n=3$ is illustrated in Figs. 1(a) and 1(b), where $n$ is the number of inserted $\mathrm{O}$ atoms. The hatched atom is emitted and goes elsewhere. The emission generates DBs at the interface when $n$ is smaller than 5 . When six $\mathrm{O}$ atoms are introduced, these DBs are perfectly terminated by the formation of a $\mathrm{Si}-\mathrm{O}-\mathrm{Si}$ or Ge-O-Ge bond, as shown in Fig. 1(c). By comparing the total-energy difference between the atomic configurations before and after the emission, the energy advantage can be defined as $E_{n o n}(n)-\left[E_{\text {emi }}(n)+\mu\right]$, where $E_{n o n}(n)$ and $E_{\text {emi }}(n)$ are the total energies of the nonemitted and emitted structures with $n \mathrm{O}$ atoms inserted, respectively, and $\mu$ is the chemical potential of Ge or $\mathrm{Si}$ in the bulk phase. The energy advantages of $\mathrm{Ge}$ and $\mathrm{Si}$ as a function of the number of inserted $\mathrm{O}$ atoms are shown in Fig. 2. When the number of inserted $\mathrm{O}$ atoms is 1 , the energy advantage of Si for the atom emission is smaller than that of Ge because the formation energy of a monovacancy is significantly larger in $\mathrm{Si}(\sim 3.3 \mathrm{eV})^{35)}$ than in $\mathrm{Ge}(\sim 1.9 \mathrm{eV}){ }^{36)}$ However, the energy advantage of Si-atom emission increases more rapidly as the number of inserted $\mathrm{O}$ atoms increases and becomes greater than that of Ge. The Si-atom emission from the surface model is preferred when $n$ is larger than 3, while the Ge atom is not emitted until the number of inserted $\mathrm{O}$ atoms becomes 6. As a further oxidation model, Figs. 1(d) and 1(e) show examples of the interface models. The energy advantages for the atom emission from the interface are shown in Fig. 3, indicating the lower energy advantage of Ge than that of Si.

These results indicate that the Ge atom preferentially remains in the substrate during the oxidation. In addition, the emitted atoms might be the source of self-interstitials, resulting in the creation of these interstitials at the interface or in the substrate. These interstitials cause the degradation of the MOS device, such as leakage current and scattering centers of inversion carriers. Because the formation energy of the Si self-interstitials $(\sim 3.5 \mathrm{eV})$ is almost equal to that of Ge self-interstitials, ${ }^{37,38)}$ the high energy advantage of the Si-atom emission causes the generation of the self-interstitials at the interface. Moreover, the trap density of the $\mathrm{Ge} / \mathrm{GeO}_{2}$ 

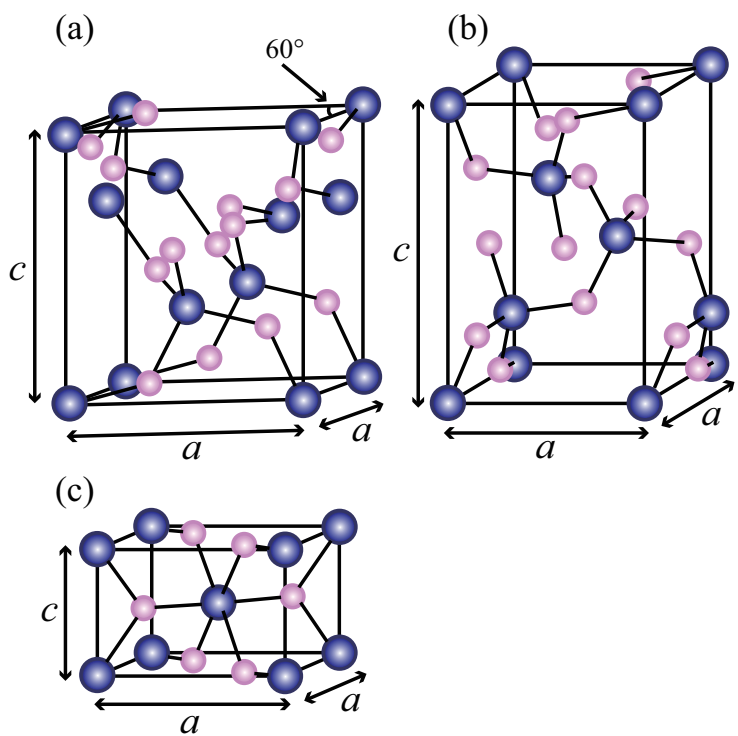

Fig. 4. (Color online) Unit cells of (a) quartz, (b) cristobalite, and (c) rutile. Pink (light) and blue (dark) balls are $\mathrm{O}$ and $\mathrm{Ge}(\mathrm{Si})$ atoms, respectively.

Table I. Calculated and experimental lattice constants (in $\AA$ ).

\begin{tabular}{lcc}
\hline & $a$ & $c$ \\
\hline $\mathrm{q}-\mathrm{GeO}_{2}$ & 4.897 & 5.636 \\
$\mathrm{c}-\mathrm{GeO}_{2}$ & 4.818 & 7.128 \\
$\mathrm{r}-\mathrm{GeO}_{2}$ & 4.418 & 2.886 \\
$\mathrm{q}-\mathrm{SiO}_{2}$ & 4.850 & 5.348 \\
$\mathrm{c}-\mathrm{SiO}_{2}$ & 4.925 & 6.828 \\
$\mathrm{r}-\mathrm{SiO}_{2}$ & 4.147 & 2.662 \\
\hline
\end{tabular}

interface becomes lower than that of the $\mathrm{Si} / \mathrm{SiO}_{2}$ interface, since the events creating volatile GeOs, the DBs, and self-interstitials at the interface rarely occur.

\subsection{Atomic structure of oxides under interface stress}

It is of interest to explore the reason why the emission probability from the $\mathrm{Ge} / \mathrm{GeO}_{2}$ interface is smaller than that from the $\mathrm{Si} / \mathrm{SiO}_{2}$ interface. One of the intuitive possibilities is the difference in the lattice-constant mismatch between the substrate and the oxide. When $\mathrm{O}$ atoms are simply inserted between Ge-Ge bonding networks, cristobalite $\mathrm{GeO}_{2}$ is formed. However, the bonding networks attain a lattice-constant mismatch of $21 \%$, which is almost the same as that between $\mathrm{Si}$ and $\mathrm{SiO}_{2}$.

In view of the chemical trend of materials, $\mathrm{SiO}_{2}$ prefers the quartz phase, in which a 

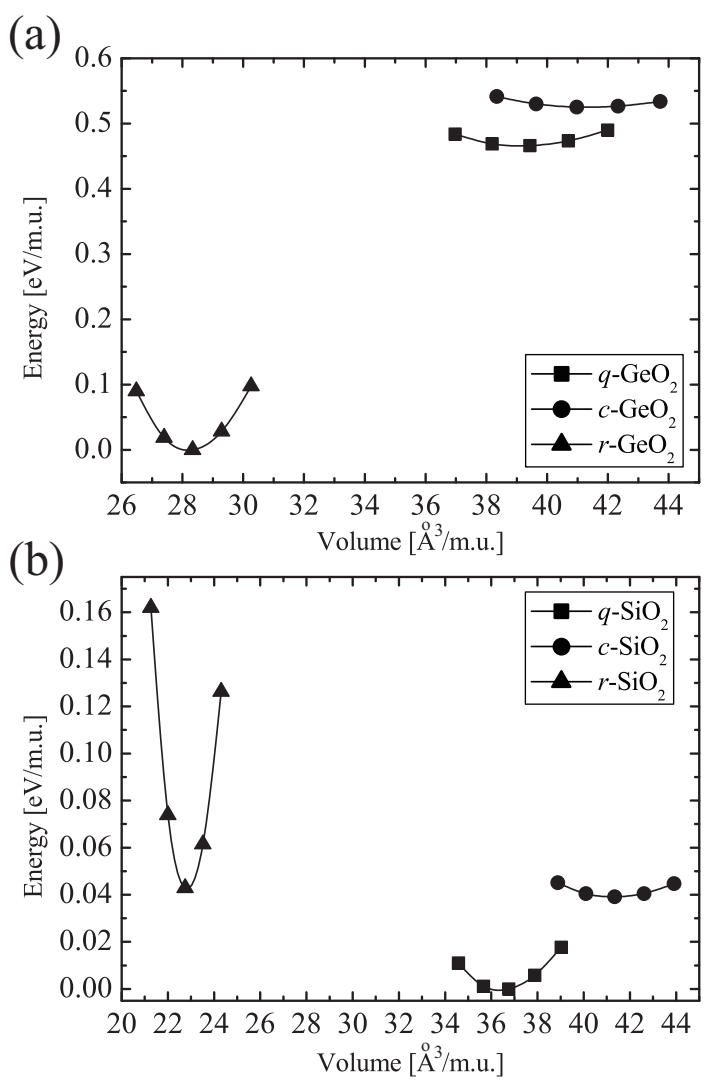

Fig. 5. Total energy per molecular unit (m.u.) as a function of volume for all polymorphs of (a) $\mathrm{GeO}_{2}$ and (b) $\mathrm{SiO}_{2}$. The zeros of energies are the rutile phase for $\mathrm{GeO}_{2}$ and the quartz phase for $\mathrm{SiO}_{2}$. Reprinted from Ref. 40.

Table II. Bond lengths $l_{i}$ (in $\AA$ ), bond angles $\theta_{j}$, and tilt angles $\delta$ (in $\operatorname{deg}$ ) of $\mathrm{GeO}_{2}$ and $\mathrm{SiO}_{2}$ polymorphs. $\theta_{1}$, $\theta_{2}, \theta_{3}$, and $\theta_{4}$ are the O-x-O angles, where $\mathrm{x}$ represents a Ge or Si atom. $l_{i}$ and $\theta_{j}$ are assigned according to magnitude.

\begin{tabular}{lccccccc}
\hline & $l_{1}$ & $l_{2}$ & $\theta_{1}$ & $\theta_{2}$ & $\theta_{3}$ & $\theta_{4}$ & $\delta$ \\
\hline $\mathrm{q}-\mathrm{GeO}_{2}$ & 1.763 & 1.755 & 114.13 & 110.69 & 107.28 & 105.39 & 29.66 \\
$\mathrm{c}-\mathrm{GeO}_{2}$ & 1.760 & 1.760 & 120.69 & 111.39 & 109.95 & 101.72 & 35.64 \\
$\mathrm{r}-\mathrm{GeO}_{2}$ & 1.918 & 1.887 & 80.25 & & & & \\
$\mathrm{q}-\mathrm{SiO}_{2}$ & 1.608 & 1.603 & 110.58 & 109.37 & 109.23 & 108.55 & 17.85 \\
$\mathrm{c}-\mathrm{SiO}_{2}$ & 1.604 & 1.603 & 111.46 & 110.02 & 109.01 & 108.15 & 25.41 \\
$\mathrm{r}-\mathrm{SiO}_{2}$ & 1.786 & 1.751 & 81.02 & & & & \\
\hline
\end{tabular}

Si atom shows fourfold coordination, while rutile $\mathrm{GeO}_{2}$, in which a $\mathrm{Ge}$ atom shows sixfold coordination, has the lowest energy among $\mathrm{GeO}_{2}$ polymorphs. ${ }^{39)}$ Detailed analyses of the structural variations of three $\mathrm{GeO}_{2}$ and $\mathrm{SiO}_{2}$ polymorphs, $\alpha$-quartz, $\alpha$-cristobalite, and rutile, under compression and expansion pressure are conducted. ${ }^{40)}$ Here and hereafter, we abbre- 

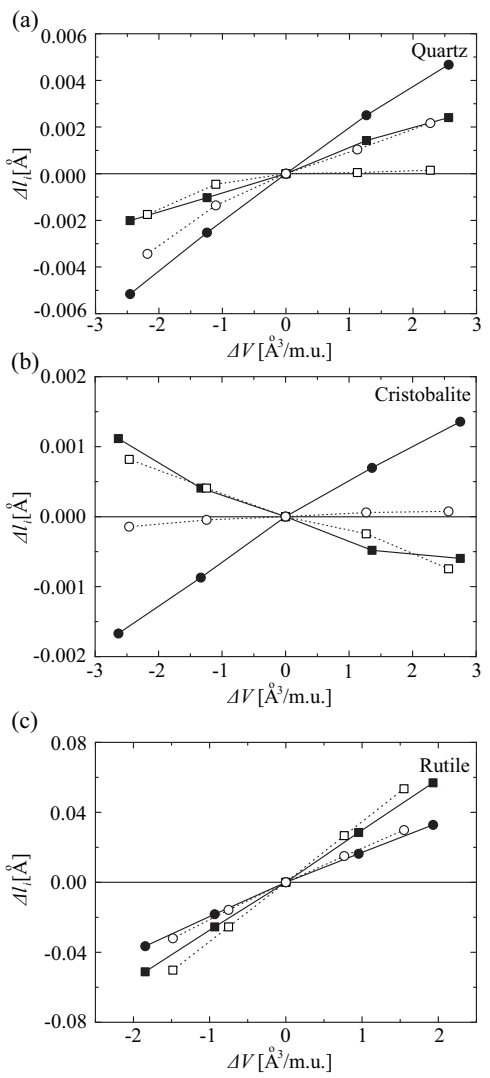
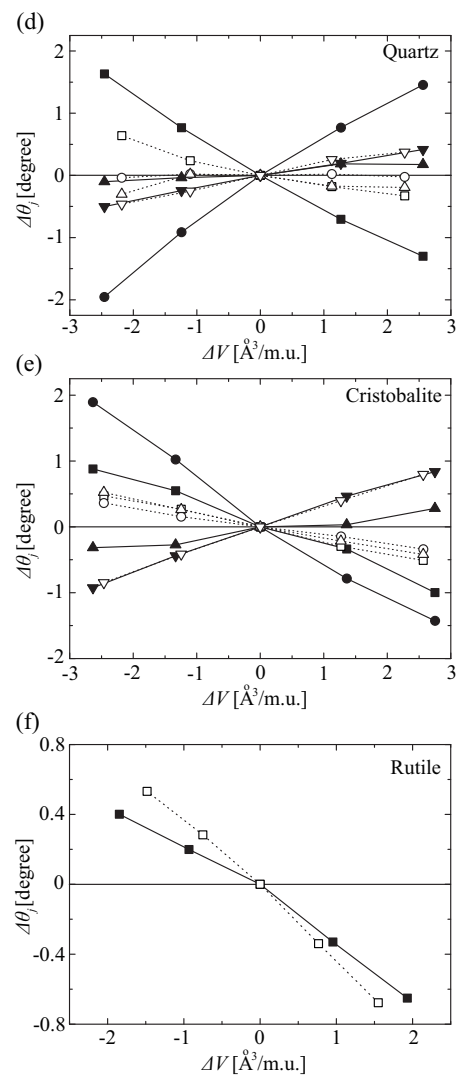
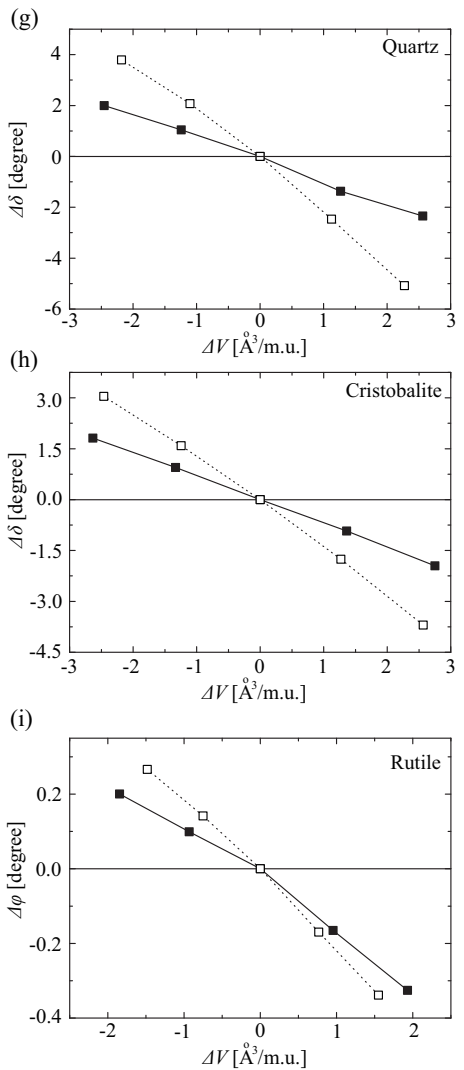

Fig. 6. Variations of $x-O$ bond lengths $\Delta l_{i}$ in (a) quartz, (b) cristobalite, and (c) rutile phases from their equilibrium points. $\Delta l_{1}$ and $\Delta l_{2}$ correspond to squares and circles, respectively. Variations of $\mathrm{O}-\mathrm{x}-\mathrm{O}$ bond angles $\Delta \theta_{j}$ in (d) quartz, (e) cristobalite, and (f) rutile phases. $\Delta \theta_{1}, \Delta \theta_{2}, \Delta \theta_{3}$, and $\Delta \theta_{4}$ correspond to squares, circles, upper triangles, and lower triangles, respectively. Variations of $\mathrm{x}-\mathrm{O}$-x bond angles $\Delta \delta$ in $(\mathrm{g})$ quartz and (h) cristobalite phases. Variations of $\mathrm{x}-\mathrm{O}-\mathrm{x}$ bond angles $\Delta \varphi$ in (i) rutile phase. $\mathrm{x}$ represents a Ge or $\mathrm{Si}$ atom. Black (white) symbols are the results of $\mathrm{GeO}_{2}\left(\mathrm{SiO}_{2}\right)$ and lines are only eye guides. Reprinted with modification from Ref. 40.

viate $\alpha$-quartz $\mathrm{GeO}_{2}, \alpha$-cristobalite $\mathrm{GeO}_{2}$, and rutile $\mathrm{GeO}_{2}$ as q- $\mathrm{GeO}_{2}, \mathrm{c}-\mathrm{GeO}_{2}$, and r- $\mathrm{GeO}_{2}$, respectively. The same abbreviations are applied for $\mathrm{SiO}_{2}$ polymorphs. $\mathrm{Ge}$ or $\mathrm{Si}$ atoms in the quartz and cristobalite phases are surrounded by four $\mathrm{O}$ atoms while those in the rutile phase is surrounded by six $\mathrm{O}$ atoms with distorted octahedral coordination, as shown in Fig. 4. The total energies per molecular unit as a function of volume for the three phases are shown in Fig. 5, in which the zeros of energy are the rutile phase for $\mathrm{GeO}_{2}$ and the quartz phase for $\mathrm{SiO}_{2}$. In the periodic table, $\mathrm{Ge}$ is between $\mathrm{Si}$ and $\mathrm{Sn}$, which is also a group IV element and is a metal. In addition, under ambient conditions, $\mathrm{SnO}_{2}$ crystallizes in the rutile phase, ${ }^{41)}$ where $\mathrm{d}$ bands of metal atoms lie below the Fermi level. ${ }^{42)}$ Since $3 \mathrm{~d}$ orbitals of Ge are occupied, $\mathrm{GeO}_{2}$ forms the sixfold rutile phase more preferentially than $\mathrm{SiO}_{2}$.

The calculated lattice constants, bond lengths $l_{i}$, bond angles $\theta_{j}$, and tilt angles $\delta$ of $\mathrm{GeO}_{2}$ 
and $\mathrm{SiO}_{2}$ are listed in Tables I and II. From the viewpoint of atomic geometry, there are two distinct $\mathrm{Ge}-\mathrm{O}(\mathrm{Si}-\mathrm{O})$ bond lengths in the $\mathrm{GeO}_{4}\left(\mathrm{SiO}_{4}\right)$ tetrahedron. In addition, $\theta_{j}$ are the O$\mathrm{Ge}-\mathrm{O}(\mathrm{O}-\mathrm{Si}-\mathrm{O})$ bond angle, ${ }^{43,44)}$ and the tilt angle is related to the Ge-O-Ge ( $\left.\mathrm{Si}-\mathrm{O}-\mathrm{Si}\right)$ bond angle. The deviations of the $\mathrm{O}-\mathrm{Ge}-\mathrm{O}$ bond angles from the ideal tetrahedral angle $\left(109.5^{\circ}\right)$ are found to be larger than those for $\mathrm{O}-\mathrm{Si}-\mathrm{O}$, which results in a distorted $\mathrm{GeO}_{4}$ tetrahedron.

The lattice-constant mismatch between $\mathrm{Ge}(\mathrm{Si})$ and its oxide induces the compressive in-plane stress at the $\mathrm{Ge} / \mathrm{GeO}_{2}\left(\mathrm{Si} / \mathrm{SiO}_{2}\right)$ interface. Since it is found by X-ray photoelectron spectroscopy that the thermal oxidation mainly generates the fourfold structure, ${ }^{45,46)}$ the bond structures of crystalline quartz and cristobalite phases are examined under the assumption that the oxidation mechanism of $\mathrm{Ge}$ is the same as that of $\mathrm{Si}$. The variations of the $\mathrm{Ge}-\mathrm{O}(\mathrm{Si}-\mathrm{O})$ bond lengths, the $\mathrm{O}-\mathrm{Ge}-\mathrm{O}(\mathrm{O}-\mathrm{Si}-\mathrm{O})$ bond angles, and the tilt angles with respect to the volume are shown in Fig. 6. The variation of the tilt angles in the rutile phase is expressed as $\Delta \varphi$ because there are no tetrahedrons in the rutile phase. It is found that the variations of the bond lengths are quite small and less than $\sim 0.1 \%$ even when the lattice constants are increased by $1 \%$. This result indicates that the bond angles are important properties in the compression or expansion of the fourfold structure. When $\mathrm{GeO}_{2}$ is compared with $\mathrm{SiO}_{2}$, the variations of the bond angles $\theta_{j}$ in $\mathrm{GeO}_{2}$ are larger than those in $\mathrm{SiO}_{2}$ [Figs. 6(d) and 6(e)], while the tilt angles $\delta$ in $\mathrm{SiO}_{2}$ vary more significantly than those in $\mathrm{GeO}_{2}$ [Figs. 6(g) and 6(h)]. When the stiffness of the tilt angle around the $\mathrm{O}$ atom is assumed to be comparable between $\mathrm{SiO}_{2}$ and $\mathrm{GeO}_{2}$, this result implies that the $\mathrm{O}-\mathrm{Si}-\mathrm{O}$ bonds are more rigid than the $\mathrm{O}-\mathrm{Ge}-\mathrm{O}$ bonds. The experimental study also reported that the $\mathrm{O}-\mathrm{Ge}-\mathrm{O}$ bond angles markedly change in $\mathrm{q}-\mathrm{GeO}_{2}$, while the tilt angle in $\mathrm{q}-\mathrm{SiO}_{2}$ varies significantly as the pressure increases. ${ }^{47)}$ These structural properties under compression or expansion agree well with the experimental result. Such chemical trend of $\mathrm{Ge}$ in the periodic table is also attributed to the distorted $\mathrm{GeO}_{4}$ tetrahedron and the variation of the $\mathrm{O}-\mathrm{Ge}-\mathrm{O}$ bond angles from the ideal tetrahedral angle. These structural properties of the $\mathrm{O}-\mathrm{Ge}-\mathrm{O}$ bonds contribute to the reduction in the interface stress during oxidation compared with its Si counterpart, resulting in the low emission probability from the $\mathrm{Ge} / \mathrm{GeO}_{2}$ interface.

\subsection{Atomic structure of oxides above the substrate}

Although the stress at the $\mathrm{Ge} / \mathrm{GeO}_{2}$ interface is expected to be small, it has to be released somewhere from the macroscopic viewpoint. Then, the atomic structures of $\mathrm{GeO}_{2}$ and $\mathrm{SiO}_{2}$ bulks in the cristobalite phases $\left(\mathrm{c}-\mathrm{GeO}_{2}\right.$ and c-SiO$\left.{ }_{2}\right)$ under pressure along the $a$-axis are investigated because these structures correspond to the directions parallel to the interface when the oxides are piled on the (001) surface. ${ }^{20)}$ Figure 7 (a) shows the initial atomic configuration, 
in which the $\mathrm{c}-\mathrm{GeO}_{2}\left(\mathrm{c}-\mathrm{SiO}_{2}\right)$ structure illustrated is tetragonal. The length of the $a$-axis from the equilibrium lattice constants, $a_{0}^{\mathrm{GeO}_{2}}\left(=4.818 \AA\right.$ ) and $a_{0}^{\mathrm{SiO}_{2}}(=4.925 \AA)$ for $\mathrm{GeO}_{2}$ and $\mathrm{SiO}_{2}$, respectively, is compressed. The total energies of $\mathrm{c}-\mathrm{GeO}_{2}$ and $\mathrm{c}-\mathrm{SiO}_{2}$ per molecular unit as a function of volume are shown in Figs. 8(a) and 8(b), respectively. The zeros of energy are the rutile phase for $\mathrm{GeO}_{2}$ and the quartz phase for $\mathrm{SiO}_{2}$. For comparison, the energy minima of other phases (quartz, cristobalite, tridymite, and rutile) without any constraints are also plotted. The $\mathrm{c}-\mathrm{GeO}_{2}$ shows a local minimum at about $0.78 a_{0}^{\mathrm{GeO} 2}$. The $\mathrm{c}-\mathrm{GeO}_{2}$ under a certain pressure transforms into a sixfold structure as shown in Fig. 7(b), which is distinct from the rutile phase, by rotating $\mathrm{O}$ atoms around the $\mathrm{Ge}$ atoms. The energy minimum of sixfold $\mathrm{GeO}_{2}$ is lower than that of fourfold $\mathrm{GeO}_{2}$ since the zero-temperature phase of $\mathrm{GeO}_{2}$ has a sixfold rutile phase as discussed in Sect. 2.2. The arrow on the upper horizontal axis corresponds to the lateral length of the $\mathrm{Ge}(001)-(1 \times 1)$ surface. Note that $\mathrm{GeO}_{2}$ forms a sixfold structure when the length of the $a$-axis is equal to that of the $(1 \times 1)$ surface, while the $\mathrm{c}^{-\mathrm{SiO}_{2}}$ still maintains a fourfold structure.

Since the lattice-constant mismatch between the sixfold $\mathrm{GeO}_{2}$ and $\mathrm{Ge}(001)$ surfaces is small $(\sim 5 \%)$, the interface stress may be released by partially forming $r-\mathrm{GeO}_{2}$ at the interface. Therefore, it is of interest to compare the energetic stability of the sixfold $\mathrm{GeO}_{2} / \mathrm{Ge}(001)$ interface with the fourfold one. Computational models are shown in Fig. 9. To set up the interface models, $\mathrm{O}$ atoms are sequentially inserted between $\mathrm{Ge}-\mathrm{Ge}$ ( $\mathrm{Si}-\mathrm{Si}$ ) bonds from the (001) surface as we assumed the layer-by-layer oxide growth in Sect. 2.1. The sixfold structure is formed by rotating the four $\mathrm{O}$ atoms around one $\mathrm{Ge}$ atom in a $\mathrm{Ge}(001)-(1 \times 1)$ surface unit. The sixfold $\mathrm{GeO}_{2} / \mathrm{Ge}$ interface is more stable by $1.92 \mathrm{eV}$ per $\mathrm{Ge}(001)-(\sqrt{2} \times \sqrt{2})$ than the fourfold one, while the fourfold $\mathrm{c}-\mathrm{SiO}_{2} / \mathrm{Si}$ interface model is preferable by $1.02 \mathrm{eV}$ compared with the sixfold one. The interface stress between $\mathrm{GeO}_{2}$ and $\mathrm{Ge}$ is released by the phase transition into the dense sixfold structure. These results indicate that the sixfold structure contributes to the release of interface stress due to the lattice-constant mismatch between the $\mathrm{c}-\mathrm{GeO}_{2}$ and $\mathrm{Ge}(001)$ surfaces as well as the small possibility of the Ge-atom emission from the interface during oxidation.

Since the lateral length of the $\operatorname{Ge}(001)-(1 \times 1)$ surface is longer than that of the sixfold $\mathrm{GeO}_{2}$ surface but shorter than that of the fourfold c- $-\mathrm{GeO}_{2}$ surface in Fig. 8(a), c- $\mathrm{GeO}_{2}$ on the $\mathrm{Ge}(001)$ surface might be composed of a mixed fourfold and sixfold structure. Figure 10 shows the computational model, in which the supercell is the doubling of the $\operatorname{Ge}(001)-(\sqrt{2} \times$ $\sqrt{2})$ surface unit in two directions. One and five neighboring $(1 \times 1)$ Ge surface units are replaced by the sixfold structure so that 12.5 and $62.5 \%$, respectively, of the $\mathrm{Ge}(001)-(1 \times$ 


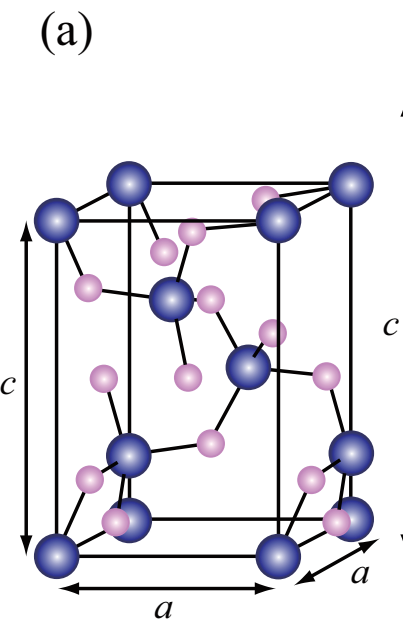

(b)

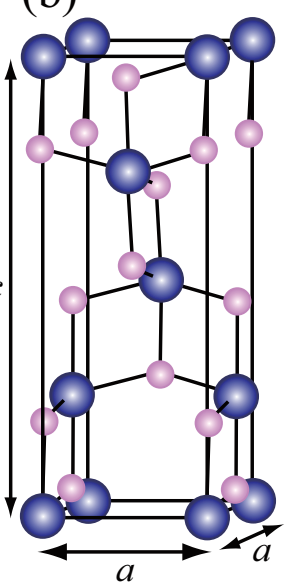

Fig. 7. (Color online) Unit cells of (a) fourfold and (b) sixfold c- $-\mathrm{GeO}_{2}$. The solid cube represents the unit-cell volume, and the blue (dark) and pink (light) balls are $\mathrm{Ge}$ and $\mathrm{O}$ atoms, respectively.

1) units are composed of the sixfold structure. The mixed interface with the $12.5 \%$ sixfold structure is even more unstable than the fully fourfold $\mathrm{GeO}_{2} / \mathrm{Ge}$ interface by $0.92 \mathrm{eV}$, although that with the $62.5 \%$ sixfold structure is more stable by $0.49 \mathrm{eV}$. The domain boundary causes the instabilities of the mixed structures; the $c$-axis of the fourfold-structure region is more than 5\% longer than that of the sixfold one. Therefore, the sixfold-structure region can exist as a large domain at the $\mathrm{GeO}_{2} / \mathrm{Ge}$ interface so that the domain boundary is minimized.

\section{Electronic structure of the defects at the interface and its contribution to leakage current}

\subsection{Dangling bond states at the interface}

Since several experimental and theoretical studies have proved that the interface trap density in the $\mathrm{Ge} / \mathrm{GeO}_{2}$ interface is smaller than that at the $\mathrm{Si} / \mathrm{SiO}_{2}$ interface without any annealing, the future development of passivation technologies to deactivate interface defects by introducing terminators will make it possible to decrease the interface trap density in the $\mathrm{Ge} / \mathrm{GeO}_{2}$ interface further. However, experimental and theoretical studies revealed that the defects are not passivated by $\mathrm{H}_{2}$ annealing in contrast to Si technology. ${ }^{19,48,49)}$ Thus, understanding the morphology and electronic structure of interface defects is one of the key issues to develop passivation technologies.

It is well known that the $\mathrm{DB}$ states at the $\mathrm{Si} / \mathrm{SiO}_{2}$ interface can be detected by electron spin-resonance (ESR) because the defect states lie between the VBM and the CBM of $\mathrm{Si}^{50}{ }^{50}$ Afanas'ev et al. ${ }^{48)}$ found that interfacial DB defects at the $\mathrm{Ge} / \mathrm{GeO}_{2}$ interface play a different 

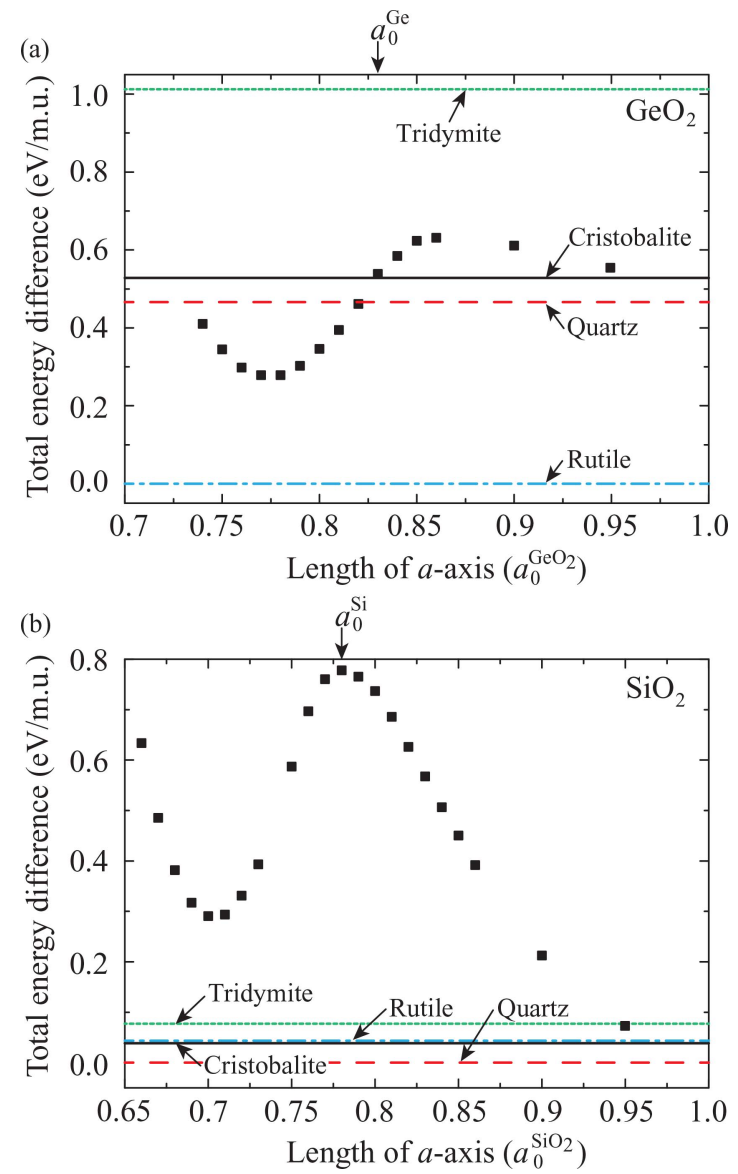

Fig. 8. (Color online) Total energy per molecular unit (m.u.) as a function of lengths of $a$-axis for (a) c-GeO2 and (b) c- $\mathrm{SiO}_{2}$. The horizontal axes correspond to the lateral lengths of $\mathrm{Ge}$ and $\mathrm{Si}(001)-(1 \times 1)$ surfaces in $a_{0}^{\mathrm{GeO}_{2}}$ and $a_{0}^{\mathrm{SiO}_{2}}$. Total energies of quartz [red (black) dashed line], cristobalite (black solid line), tridymite [green (black) dotted line], and rutile [blue (black) long dashed short dashed line] are also shown for comparison. The zeros of energies are rutile for $\mathrm{GeO}_{2}$ and quartz for $\mathrm{SiO}_{2}$. Vertical arrows indicate lattice constants of $\mathrm{Ge}\left(a_{0}^{G e}\right)$ and $\mathrm{Si}\left(a_{0}^{S i}\right)$ bulks, respectively. Reprinted with permission from Ref. 20. Copyright 2011 Amreican Physical Society.

role from those at the $\mathrm{Si} / \mathrm{SiO}_{2}$ interface using ESR; a measurable density of DBs of the semiconductor surface atoms is not found at $\mathrm{Ge} / \mathrm{GeO}_{2}$ interfaces, and the defects characterized by an isotropic signal with $g \sim 2.002-2.004$ are observed instead. However, there remains a certain amount of interface defects because the interface trap density shown in Ref. 48 was still higher than that of typical $\mathrm{Si} / \mathrm{SiO}_{2}$ interfaces after hydrogen annealing. This result implies the ESR inactivity of the $\mathrm{Ge}-\mathrm{DBs}$ at $\mathrm{Ge} / \mathrm{GeO}_{2}$ interfaces.

From the viewpoint of the electronic structure of the DBs, the position of the DB states in Ge bulk is investigated by the $G_{0} W_{0}$ approximation ${ }^{12)}$ and the hybrid functional method. ${ }^{13)}$ The former claimed that the DBs in Ge bulk have energy levels below the VBM of Ge and are 
(a)

0

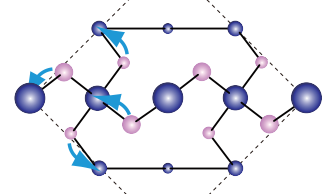

0

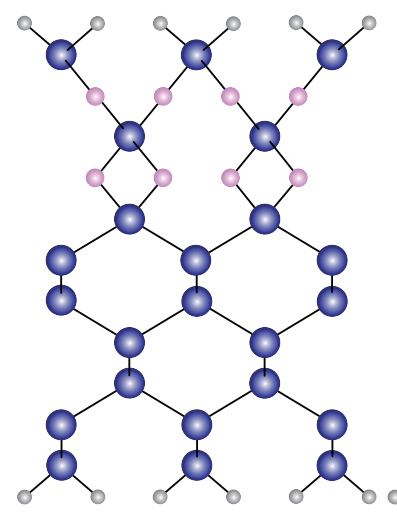

(b)

0<smiles>o1oooooooooo1</smiles>

○

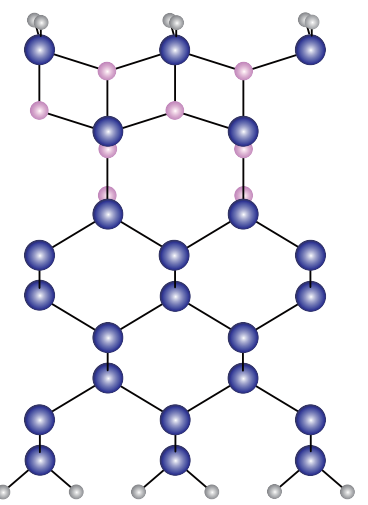

Fig. 9. (Color online) Top views and side views of (a) fourfold and (b) sixfold $\mathrm{GeO}_{2} / \mathrm{Ge}(001)$ interfaces. The blue (dark large), pink (light middle), and grey (light small) balls are $\mathrm{Ge}, \mathrm{O}$, and $\mathrm{H}$ atoms, respectively. The dotted square in the top views represents a $\operatorname{Ge}(001)-(\sqrt{2} \times \sqrt{2})$ surface unit and the arrows indicate rotational directions to transform into sixfold structures.

(a)

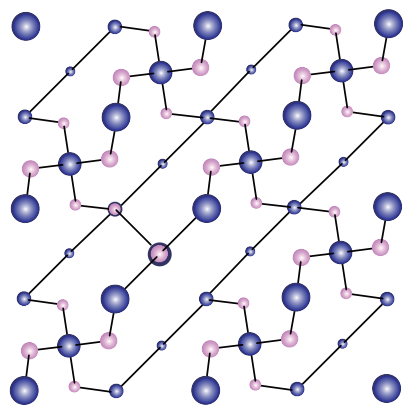

(b)

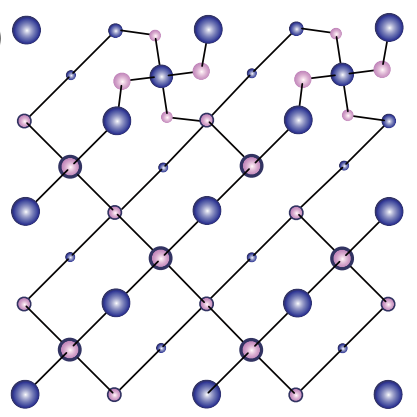

Fig. 10. (Color online) Top views of mixed fourfold and sixfold structures. (a) 12.5 and (b) $62.5 \%$ of $\mathrm{Ge}(001)-(1 \times 1)$ surface units are composed of the sixfold structures. The blue (dark) and pink (light) balls are $\mathrm{Ge}$ and $\mathrm{O}$ atoms, respectively. 


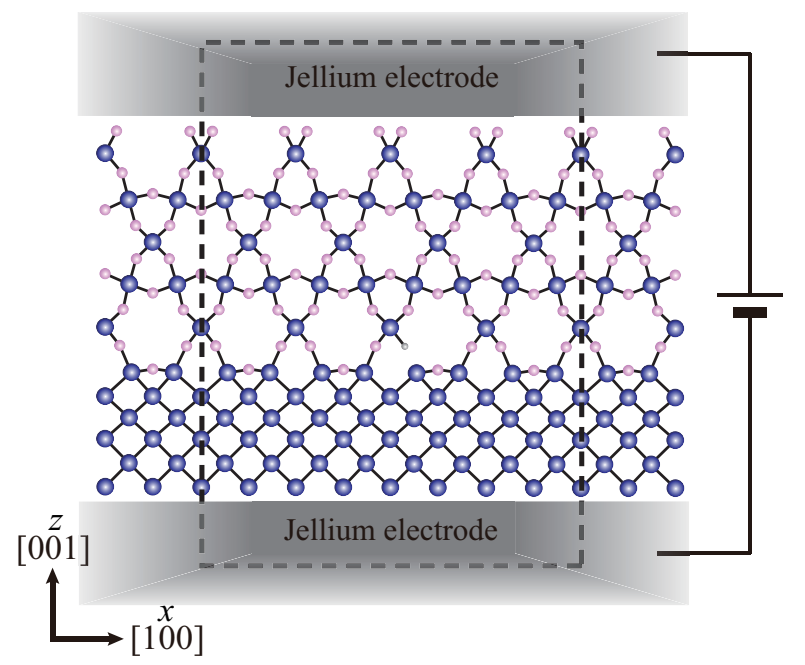

Fig. 11. (Color online) Schematic image of computational model for interface. The rectangle enclosed by broken lines represents the scattering region when the leakage current is calculated. Blue (dark large), pink (light middle), and gray (light small) balls represent $\mathrm{Ge}$ (Si), O, and $\mathrm{H}$ atoms, respectively.

therefore negatively charged. In contrast, the latter reported that the DBs in Ge bulk induce two very close defect levels lying just above the VBM within the certain range of the empirical mixing parameter of the hybrid functional method. Although these studies derive the opposite conclusions, they raised questions about the difference in the electronic structure between the Ge-DBs in Ge bulk and the Si-DBs in Si bulk, and the contribution of the interface stress caused by the lattice-constant mismatch between the semiconductor and the oxide to the position of the DB states. Here, we introduce first-principles study on the difference in the electronic structure between two interfaces ${ }^{21)}$ using the RSPACE code. The interface atomic structure, consisting of an $\alpha$-quartz crystalline oxide layer on a (001) substrate, is shown in Fig. 11. The DBs at the interface are generated by removing one $\mathrm{O}$ atom from the interface and terminating one $\mathrm{DB}$ by $\mathrm{H}$ atom.

The lattice-constant mismatch between $\mathrm{Ge}(\mathrm{Si})$ and $\mathrm{GeO}_{2}\left(\mathrm{SiO}_{2}\right)$ induces the interface reconstruction, which is not taken into account in the $\mathrm{Ge}(\mathrm{Si})$ bulk model. ${ }^{12,13)}$ The $c$-axis is slightly longer than the axis of the bulk (less than 1\%) in both the Ge and Si cases, whereas the $a$-axis of the $\alpha$-quartz oxide is $11 \%$ (9\%) longer than the corresponding axis of the $\mathrm{Ge}$ ( $\mathrm{Si}$ ) bulk. Thus, the stress acting on the interface atoms is similar for both $\mathrm{GeO}_{2}$ and $\mathrm{SiO}_{2}$. Figure 12 shows the interface atomic structure after structural optimization. The interface Ge atom with the DB is raised up while the $\mathrm{Si}$ atom is pulled down so that the $\pi$ electron forms a $P_{b}$-type defect in the $\mathrm{Si}$ substrate.

The density of states (DOS) of interfaces with the DBs is shown in Fig. 13 for further 


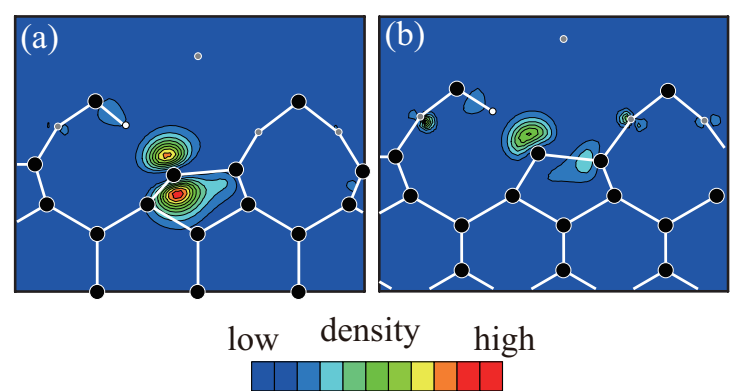

Fig. 12. (Color online) Atomic structures of interfaces and contour plots of charge density distributions of DB states. The planes shown are along the cross section in the (110) plane including DBs. (a) Si-DB state at $E_{F}$ and (b) Ge-DB state at $E_{F}-0.3 \mathrm{eV}$. The lowest contours are $6.75 \times 10^{-3} e / \AA^{3}$ and the subsequent contour lines represent values larger than $6.75 \times 10^{-3} e / \AA^{3}$. The symbol meanings are the same as those in Fig. 11 . Reprinted from Ref. 21.

investigation of the relationship between the atomic configuration and the electronic structure. Figure 13 is plotted by integrating the DOS along the plane parallel to the interface, $\rho(z, E)=$ $\int|\psi(\vec{r}, E)|^{2} d \vec{r}_{\|}$, where $\vec{r}=(x, y, z), \vec{r}_{\|}=(x, y), \psi$ is the wave function, and $E$ is the energy of the states. Note that the energy band gap of Si and Ge substrates is underestimated owing to the usage of the local density approximation. ${ }^{51)}$ The states accumulating at $E_{F}-1.0 \mathrm{eV}$ and indicated by the red downward arrows in Fig. 13(c) [Fig. 13(a)] are attributed to the Ge(Si-) $\mathrm{O}$ bonds, where $E_{F}$ is the Fermi level, since the charge density distributions accumulate between the $\mathrm{Ge}-\left(\mathrm{Si}^{-}\right)$and $\mathrm{O}$-atom layers. In the case of $\mathrm{Si}$, when the $\mathrm{DB}$ is introduced in the $\mathrm{Si} / \mathrm{SiO}_{2}$ interface, the DOS at $E_{F}-1.0 \mathrm{eV}$ decreases and the state appears in the energy band gap, indicated by the white downward arrows in Fig. 13(b). On the other hand, the peak of the DOS at $E_{F}-1.0 \mathrm{eV}$ related to the Ge-O bonds becomes shallow, and the DOS at $E_{F}-0.3 \mathrm{eV}$, denoted by the white downward arrows in Fig. 13(d), increases in the case of the $\mathrm{Ge} / \mathrm{GeO}_{2}$ interface. In the periodic table, Ge exists between $\mathrm{Si}$ and $\mathrm{Sn}$, the latter being metallic. The Ge-O bonds at the interface do not have a strongly preferential $\mathrm{sp}^{3}$-bonding direction. ${ }^{11)}$ Thus, the bonding network of $\mathrm{Ge} / \mathrm{GeO}_{2}$ is easily deformed from the $\mathrm{sp}^{3}$ structure and exhibits more metallic properties than that of $\mathrm{Si} / \mathrm{SiO}_{2}$. In addition, the band gap of the diamond structure of group IV elements is formed by the energy difference between $\mathrm{sp}^{3}$ bonding and antibonding states, and the energy gain due to the formation of covalent bonds in Ge is smaller than those in Si. These characteristics of Ge and the larger offset of the VBM than that of the CBM at the interface ${ }^{20,26)}$ contribute to keeping the DB states below the VBM of Ge. Moreover, Haneman ${ }^{52)}$ reported that the $\mathrm{sp}^{3} \mathrm{DB}$ on the Si surface is stabilized owing to the strong contribution of the s electron when the atom with a DB is raised up. The 

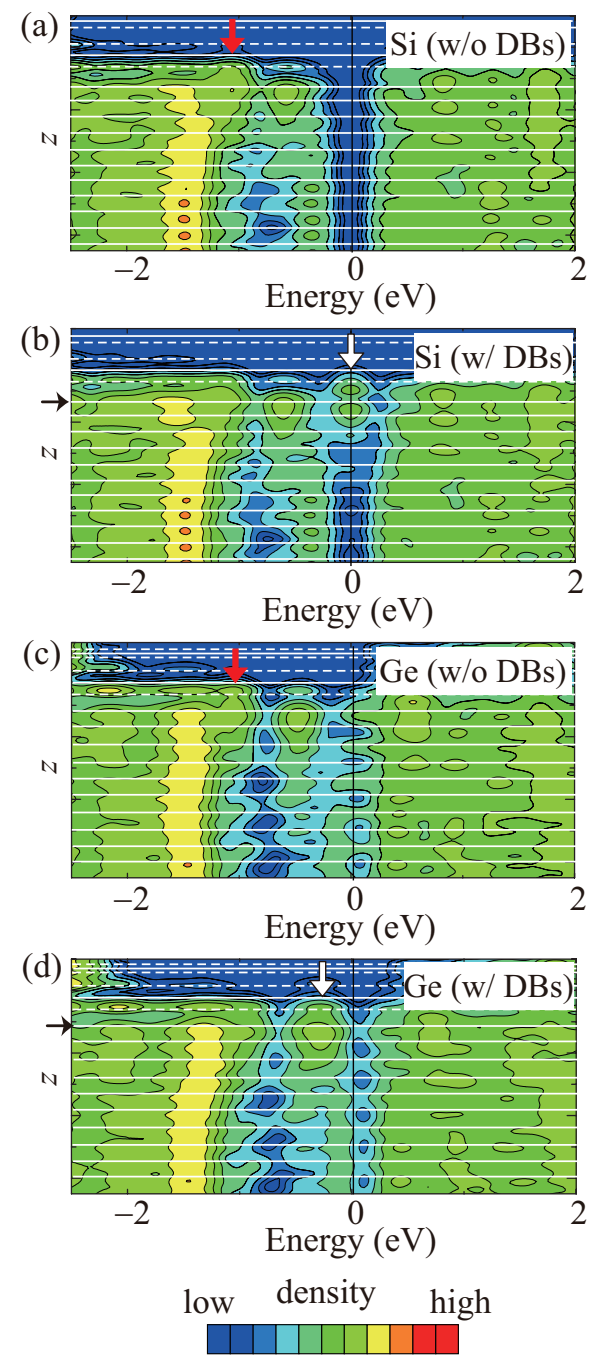

Fig. 13. (Color online) Distributions of DOS integrated on a plane parallel to the interface as functions of relative energy from the Fermi level. (a) $\mathrm{Si} / \mathrm{SiO}_{2}$ without $\mathrm{DBs}$, (b) $\mathrm{Si} / \mathrm{SiO}_{2}$ with $\mathrm{DBs}$, (c) $\mathrm{Ge} / \mathrm{GeO}_{2}$ without DBs, and (d) $\mathrm{Ge} / \mathrm{GeO}_{2}$ with DBs. The zeros of energies are chosen to be the Fermi level. Each contour represents twice or half the density of the adjacent contour lines, and the lowest contour is $1.45 \times 10^{-4} e / \mathrm{eV} / \AA$. The solid and dashed lines represent the vertical positions of $\mathrm{Si}(\mathrm{Ge})$ and $\mathrm{O}$ atomic layers, respectively. The right arrows denote the positions of the Si and Ge atoms forming DBs. Reprinted from Ref. 21.

relationship between the position of the DB states and the atomic configuration in this study is consistent with the model proposed by Heneman. Therefore, the significant difference in the ESR signal from Ge-DBs ${ }^{48}$ is caused by the difference in the electronic structures and atomic configurations from $\mathrm{Si}$-DBs.

Later, Houssa et al. ${ }^{49)}$ claimed that the ESR signal observed at $\mathrm{Ge} / \mathrm{GeO}_{2}$ interfaces could be assigned to a DB at a Ge atom backbonded to two Ge atoms and one $\mathrm{O}$ atom, which does not contradict the results obtained by the above-mentioned electronic-structure calculation. 
Chang et al. ${ }^{22)}$ also investigated the Ge-DBs with different $\mathrm{O}$ backbonds at $\mathrm{Ge} / \mathrm{GeO}_{2}$ interfaces and reported, on the basis of the hybrid functional method, that the state of the $P_{b}$-type defect with the Ge atom backbonded to three Ge atoms lies above the VBM. However, the mixing parameter of the hybrid functional method, which significantly affects the position of the DB state, ${ }^{18)}$ is large so that the state appears above the VBM, and thus the effect of the mixing parameter on the position of interface DB states is still controversial in the hybrid functional method.

\subsection{Gate leakage property of the metal-oxide-semiconductor structure}

In the case of the $\mathrm{Si} / \mathrm{SiO}_{2}$ interface, the $P_{b}$-type defect increases the leakage current. ${ }^{53)}$ Since the state attributed to the Ge-DB does not lie inside the band gap of Ge, it is interesting to determine whether the leakage current is increased by the presence of the Ge-DBs at the interface. The recent evolution of the computational technique of the first-principles electron-transport calculation enables us to investigate the relationship between the interface defects and the leakage current. ${ }^{54,55)}$ Applicability to first-principles electron-transport calculation $^{31,56,57)}$ is one of the advantages of the real-space finite-difference method ${ }^{31,58,59)}$ over the other first-principles calculation methods, e.g., plane-wave expansion ${ }^{60)}$ or linear combination of atomic orbitals. ${ }^{61,62)}$ The computational model for the transport calculation is illustrated in Fig. 11. The MOS interface is sandwiched between the metallic electrodes, and the conductance for the electrons coming from the lower electrodes to the upper one is calculated, assuming that electrons accumulate at the interface by gate bias. ${ }^{55)}$ Figure 14 shows the conductance spectra of the interface with and without the DBs. The conductance spectrum corresponds to the differentiation of the amount of leakage current with respect to the bias voltage. Although the Fermi level is determined by the work function of the electrodes, the overall feature of the local DOS at the interfaces does not change; the states of the Ge-DBs are under the VBM while those of the Si-DB lie between the VBM and the CBM. The tunneling probability for the leakage current exponentially increases with respect to the energy difference between the incident electron and the conduction band, which is the barrier height. Because the band gap of $\mathrm{GeO}_{2}$ is smaller than that of $\mathrm{SiO}_{2}$, the conductance of the $\mathrm{Ge} / \mathrm{GeO}_{2}$ interface is larger than that of the $\mathrm{Si} / \mathrm{SiO}_{2}$ interface. When the $\mathrm{DB}$ is introduced to the $\mathrm{Si} / \mathrm{SiO}_{2}$ interface, the sharp peak due to the contribution of the DB appears in the conductance spectrum. On the other hand, the significant contribution of the Ge-DB states is not observed in the case of the $\mathrm{Ge} / \mathrm{GeO}_{2}$ interface. This is because the Ge-DB states spread energetically and are coupled with the states of the substrates, resulting in the shallow peak in the conductance 

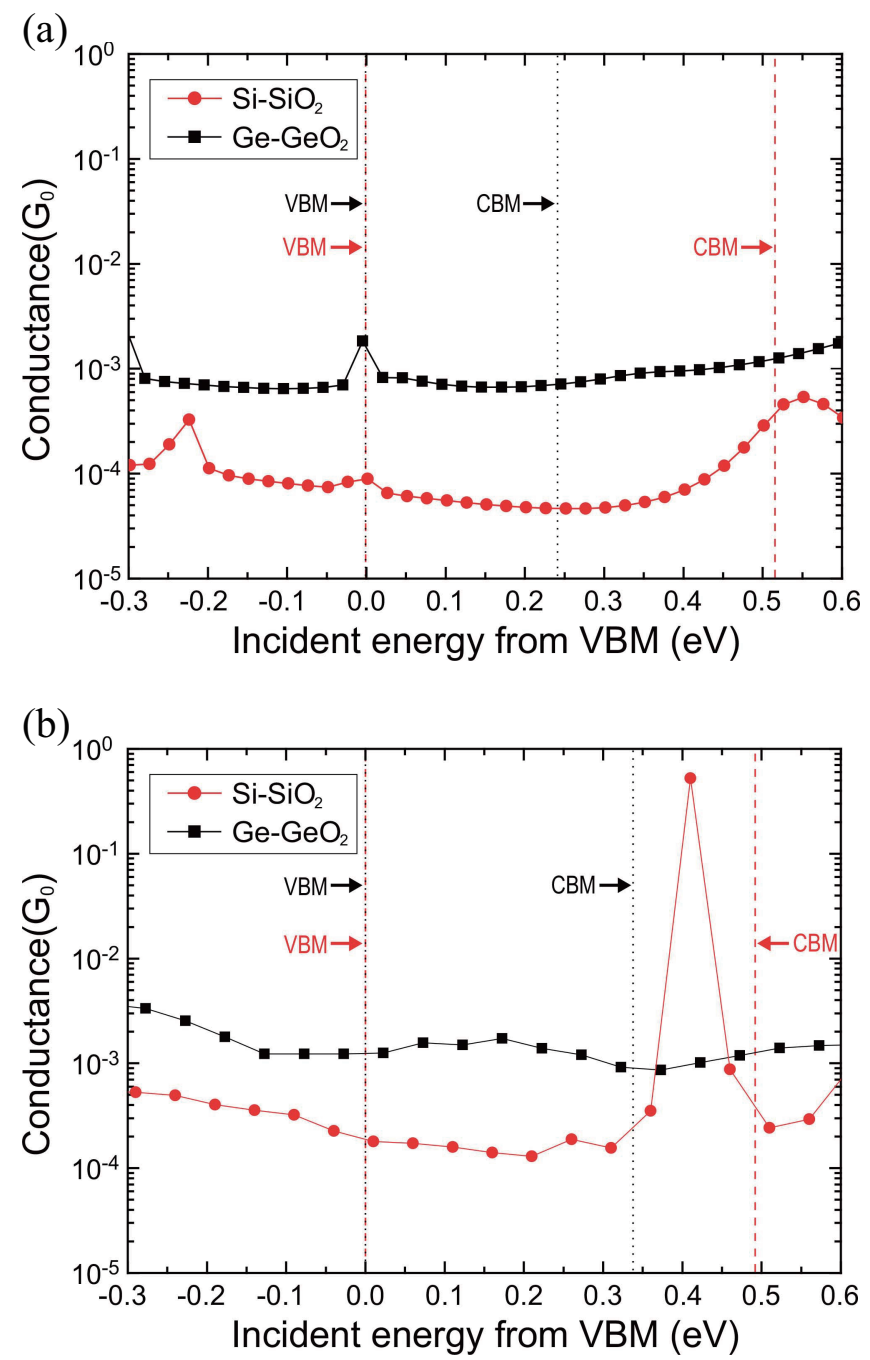

Fig. 14. (Color online) Conductance of the $\mathrm{Si} / \mathrm{SiO}_{2}$ and $\mathrm{Ge} / \mathrm{GeO}_{2}$ interfaces (a) without and (b) with $\mathrm{DBs}$ as a function of incident electron energy measured from the VBM of the substrates. The (red) circles and (black) squares represent conductance spectra of the $\mathrm{Si} / \mathrm{SiO}_{2}$ and $\mathrm{Ge} / \mathrm{GeO}_{2}$ interfaces, respectively. The VBM and $\mathrm{CBM}$ of the $\mathrm{Si}(\mathrm{Ge})$ substrate are indicated by dashed (dotted) vertical lines. Reprinted with permission from Ref. 55. Copyright 2015 Amreican Physical Society

spectrum. Therefore, it can be concluded that the contribution of the Ge-DBs at the interface to the leakage current as well as electronic structure is different from those of the Si-DB.

\section{Summary}

We have reviewed a series of first-principles studies on the atomic and electronic structures of the $\mathrm{Ge} / \mathrm{GeO}_{2}$ interface. First-principles total-energy calculation for the interface atomic structures revealed that $\mathrm{Ge}$ atoms at the $\mathrm{Ge} / \mathrm{GeO}_{2}$ interface are more hardly emitted from the oxidation front than $\mathrm{Si}$ atoms at the $\mathrm{Si} / \mathrm{SiO}_{2}$ interface. This result supports the experimental result that a lower interface trap density will be realizable in the $\mathrm{Ge} / \mathrm{GeO}_{2}$ interface. Since 
Ge exists between the semiconductor Si and the metal $\mathrm{Sn}$ in the periodic table, Ge exhibits more metallic property than Si. Indeed, the sixfold rutile phase is the most stable among the phases of $\mathrm{GeO}_{2}$ while $\mathrm{SiO}_{2}$ preferentially forms the fourfold quartz structure, which is interpreted on the basis of the chemical trend because $\mathrm{SnO}_{2}$ prefers a sixfold structure. The small lattice constant of the stable sixfold structure of $\mathrm{GeO}_{2}$ suppresses the increase in the interface stress, resulting in the small probability of atom emission and the low interface trap density of the $\mathrm{Ge} / \mathrm{GeO}_{2}$ interface. The electronic-structure calculation for the defects at the $\mathrm{Ge} / \mathrm{GeO}_{2}$ interface found that the Ge-DB states lie below the VBM of Ge, indicating the ESR inactivity of the Ge-DB defects. First-principles electron-transport calculation elucidated that the states do not increase the leakage current. Therefore, even if they are created, their contribution to the leakage current is smaller than that of Si-DB. Thus, we can conclude that the Ge-DBs behave differently from the Si-DBs. These results from the first-principles calculation imply that $\mathrm{Ge}$ is the promising candidate for the channel material in next-generation devices owing to the low trap density in its interface as well as the high carrier mobility, although effective passivation techniques have to be developed to decrease the interface trap density further.

From the viewpoint of the computational investigation for device sciences, the atom emission from the oxidation front in $\mathrm{SiC} / \mathrm{SiO}_{2}$ interfaces has recently been discussed, which can be used for high-temperature and power electronic devices, under conditions where the traditionally used Si fails; first-principles calculation revealed that $\mathrm{C}$ atoms are emitted as $\mathrm{CO}$ molecules from the oxidation front. ${ }^{63)}$ Moreover, the interface defects that appear during oxidation significantly affect the electronic structure at the interface, ${ }^{64)}$ and the first-principles electron-transport calculation revealed that the carriers are scattered by the modified electronic structure at the interface. ${ }^{65)}$ Work in progress is to examine the carrier scattering at the $\mathrm{Ge} / \mathrm{GeO}_{2}$ or $\mathrm{Ge} /$ high- $k$ interface for a fuller understanding of how the scattering properties of the interface change with interface defects.

\section{Acknowledgements}

This research was partially supported by the Computational Materials Science Initiative (CMSI), by a Grant-in-Aid for Scientific Research on Innovative Areas (Grant No. 22104007), and by the Core-to-Core program of the Deutsche Forschungsgemeinschaft and the Japan Society for the Promotion of Science from the Ministry of Education, Culture, Sports, Science and Technology, Japan. The numerical calculation was carried out using the computer facilities of the Institute for Solid State Physics at the University of Tokyo and the Center for Computational Sciences at the University of Tsukuba. 


\section{References}

1) H. Fukuda, T. Ueno, H. Kawarada, and I. Ohdomari, Jpn. J. Appl. Phys., 32, L569 (1993).

2) K. Kita, S. Suzuki, H. Nomura, T. Takahashi, T. Nishimura, and A. Toriumi, Jpn. J. Appl. Phys. 47, 2349 (2008).

3) C. H. Lee, T. Nishimura, K. Nagashio, K. Kita, and A. Toriumi, Ext. Abst. Solid State Devices and Materials, 2008, p. 16.

4) C. H. Lee, T. Tabata, T. Nishimura, K. Nagashio, K. Kita, and A. Toriumi, Appl. Phys. Express 2, 071404 (2009).

5) D. Kuzum, A. J. Pethe, T. Krishnamohan, Y. Oshima, Y. Sun, J. P. McVittie, P. A. Pianetta, P. C. McIntyre, and K. C. Saraswat, IEDM Tech. Dig., 2007, p. 723.

6) H. Matsubara, T. Sasada, M. Takenaka, and S. Takagi, Appl. Phys. Lett. 93, 032104 (2008).

7) T. Hosoi, K. Kutsuki, G. Okamoto, M. Saito, T. Shimura, and H. Watanabe, Appl. Phys. Lett. 94, 202112 (2009).

8) P. Hohenberg and W. Kohn, Phys. Rev. 136, B864 (1964).

9) W. Kohn and L. J. Sham, Phys. Rev. 140, A1133 (1965).

10) M. Houssa, G. Pourtois, M. Caymax, M. Meuris, M. M. Heyns, V. V. Afanas'ev, and A. Stesmans, Appl. Phys. Lett. 93, 161909 (2008).

11) S. Saito, T. Hosoi, H. Watanabe, and T. Ono, Appl. Phys. Lett. 95, 011908 (2009).

12) J. R. Weber, A. Janotti, P. Rinke, and C. G. Van de Walle, Appl. Phys. Lett. 91, 142101 (2007).

13) P. Broqvist, A. Alkauskas, and A. Pasquarello, Phys. Rev. B 78, 075203 (2008).

14) L. Hedin, Phys. Rev. 139, A796 (1965).

15) J. P. Perdew, M. Ernzerhof, and K. Burke, J. Chem. Phys. 105, 9982 (1996).

16) G. Pourtois, M. Houssa, A. Delabie, T. Conard, M. Caymax, M. Meuris, and M. M. Heyns, Appl. Phys. Lett. 92, 032105 (2008).

17) M. Houssa, G. Pourtois, M. Caymax, M. Meuris, and M. M. Heyns, Appl. Phys. Lett. 92, 242101 (2008).

18) P. Broqvist, J. F. Binder, and A. Pasquarello, Appl. Phys. Lett. 94, 141911 (2009).

19) L. Tsetseris and S. T. Pantelides, Appl. Phys. Lett. 95, 262107 (2009).

20) S. Saito and T. Ono, Phys. Rev. B 84, 085319 (2011).

21) T. Ono and S. Saito, Appl. Phys. Express 4, 021303 (2011). 
22) H.-C. Chang, S.-C. Lu, T.-P. Chou, C.-M. Lin, and C. W. Liu, Appl. Phys. Lett. 111, 076105 (2012).

23) P. Broqvist, J. F. Binder, and A. Pasquarello, Physica B 407, 2926 (2012).

24) L. Zhang, H. Li, Y. Guo, K. Tang, J. Woicik, J. Robertson, and P. C. McIntyre, ACS Appl. Mater. Interfaces 7, 20499 (2015).

25) N. Ikarashi, K. Watanabe, and Y. Miyamoto, Phys. Rev. B 62, 15989 (2000).

26) T. Yamasaki, C. Kaneta, T. Uchiyama, T. Uda, and K. Terakura, Phys. Rev. B 63, 115314 (2001).

27) H. Kageshima and K. Shiraishi, Appl. Surf. Sci. 130, 176 (1998).

28) H. Kageshima and K. Shiraishi, Phys. Rev. Lett. 81, 5936 (1998).

29) T. Yamasaki, K. Kato, and T. Uda, Phys. Rev. Lett. 91, 146102 (2003).

30) Z. Ming, K. Nakajima, M. Suzuki, K. Kimura, M. Uematsu, K. Torii, S. Kamiyama, Y. Nara, and K. Yamada, Appl. Phys. Lett. 88, 153516 (2006).

31) K. Hirose, T. Ono, Y. Fujimoto, and S. Tsukamoto, First-Principles Calculations in Real-Space Formalism, Electronic Configurations and Transport Properties of Nanostructures (Imperial College Press, London, 2005).

32) T. Ono and K. Hirose, Phys. Rev. Lett. 82, 5016 (1999).

33) T. Ono and K. Hirose, Phys. Rev. B 72, 085115 (2005).

34) T. Ono, M. Heide, N. Atodiresei, P. Baumeister, S. Tsukamoto, and S. Blügel, Phys. Rev. B 82, 205115 (2010).

35) M. J. Puska, S. Pöykkö, M. Pesola, and R. M. Nieminen, Phys. Rev. B 58, 1318 (1998).

36) A. Fazzio, A. Janotti, A. J. R. da Silva, and R. Mota, Phys. Rev. B 61, R2401 (2000).

37) M. D. Moreira, R. H. Miwa, and P. Venezuela, Phys. Rev. B 70, 115215 (2004).

38) R. J. Needs, J. Phys.: Condens. Matter 11, 10437 (1999).

39) D. M. Christie and J. R. Chelikowsky, Phys. Rev. B 62, 14703 (2000).

40) S. Saito and T. Ono, Jpn. J. Appl. Phys. 50, 021503 (2011).

41) R. W. G. Wyckoff, Crystal Structures (Wiley, New York, 1965) Vol. 1.

42) C. E. Ekuma and D. Bagayoko, Jpn. J. Appl. Phys. 50, 101103 (2011).

43) H. Grimm and B. Dorner, J. Phys. Chem. Solids 36, 407 (1975).

44) M. O’Keefee and B. G. Hyde, Acta Crystallogr., Sect. B 32, 2923 (1976).

45) A. Molle, Md. N. K. Bhuiyan, G. Tallarida, and M. Fanciulli, Appl. Phys. Lett. 89, 083504 (2006).

46) T. Hattori and T. Suzuki, Appl. Phys. Lett. 43, 470 (1983).

47) J. D. Jorgensen, J. Appl. Phys. 49, 5473 (1978). 
48) V. V. Afanas'ev, Y. G. Fedorenko, and A. Stesmans, Appl. Phys. Lett. 87, 032107 (2005).

49) M. Houssa, G. Pourtois, V. V. Afanas'ev, and A. Stesmans, Appl. Phys. Lett. 99, 212103 (2011).

50) A. Stesmans and V. V. Afanas'ev, Appl. Phys. Lett. 77, 1469 (2000).

51) J. P. Perdew and A. Zunger, Phys. Rev. B 23, 5048 (1981).

52) D. Haneman, Phys. Rev. 121, 1093 (1961).

53) T. Ono, Phys. Rev. B 79, 195326 (2009).

54) S. Iwase, T. Hoshi, and T. Ono, Phys. Rev. E 91, 063305 (2015).

55) Y. Egami, S. Iwase, S. Tsukamoto, T. Ono and K. Hirose, Phys. Rev. E 92, 033301 (2015).

56) Y. Fujimoto and K. Hirose, Phys. Rev. B 67, 195315 (2003).

57) T. Ono and K. Hirose, Phys. Rev. B 70, 033403 (2004).

58) J. R. Chelikowsky, N. Troullier, and Y. Saad, Phys. Rev. Lett. 72, 1240 (1994).

59) J. R. Chelikowsky, N. Troullier, K. Wu, and Y. Saad, Phys. Rev. B 50, 11355 (1994).

60) M. C. Payne, M. P. Teter, D. C. Ailan, T. A. Arias and J. D. Joannopouios, Rev. Mod. Phys. 64, 1045 (1992).

61) A. Szabo and N. S. Ostlund, Modern Quantum Chemistry: Introduction to Advanced Electronic Structure Theory (Dover, New York, 1996)

62) F. Jensen, Introduction to Computational Chemistry (Wiley, Chichester, U. K., 2006) 2nd ed.

63) T. Ono and S. Saito, Appl. Phys. Lett. 106, 081601 (2015).

64) C. Kirkham and T. Ono, J. Phys. Soc. Jpn. 85, 024701 (2016).

65) S. Iwase, C. Kirkham and T. Ono, in preparation for publication. 